\title{
Racl and RhoA Promote Neurite Outgrowth through Formation and Stabilization of Growth Cone Point Contacts
}

\author{
Stephanie Woo and Timothy M. Gomez \\ Department of Anatomy and Program in Cellular and Molecular Biology, University of Wisconsin, Madison, Wisconsin 53706
}

\begin{abstract}
Growth cone advance depends on coordinated membrane protrusion and adhesion to the extracellular matrix. Although many studies have addressed the mechanisms responsible for membrane protrusion, the assembly of integrin-dependent adhesion sites known as point contacts remains poorly understood in growth cones. We show balanced Racl activity controls both leading edge protrusion and point contact dynamics during neurite outgrowth. Immunocytochemistry and live imaging of paxillin-green fluorescent protein (GFP) showed that inhibiting Racl blocked point contact formation, whereas Rac1 overactivation produced small, unstable point contacts. Both inhibition and overactivation of Racl reduced the persistence of lamellar protrusions and neurite outgrowth. Inhibition of ROCK (Rho kinase), a RhoA effector, perturbed protrusion and point contact dynamics similar to Racl overactivation. Moreover, the repulsive guidance cue Semaphorin 3A, which signals through Rac1, destabilizes point contacts. Together, our data suggest that coordinated Rho GTPase activities regulate neurite outgrowth through point contact formation and stabilization of membrane protrusion.
\end{abstract}

Key words: axon pathfinding; integrin; Rho GTPase; Semaphorin 3A; Xenopus; paxillin

\section{Introduction}

Adhesion to the extracellular matrix (ECM) is a critical step for cell spreading and regulation of cell motility, including growth cone migration during axon guidance (Suter and Forscher, 2000; Ridley et al., 2003). Integrin receptors composed of $\alpha / \beta$ heterodimers are major mediators of adhesion to ECM ligands. Integrin engagement of ECM ligands results in receptor clustering and the formation of various adhesion complexes (Geiger et al., 2001) that provide a linkage to the actin cytoskeleton and support the force generation necessary for growth cone advance (Suter et al., 1998; Beningo et al., 2001). In addition to providing adhesive support for mechanical tension, studies have shown that integrin receptors activate second-messenger signaling cascades, which help coordinate assembly and disassembly of adhesion sites (Gomez et al., 2001; Giannone et al., 2002; Ivins et al., 2004).

Work in fibroblasts has indicated an important role for the Rho GTPases RhoA and Racl in regulating integrin-dependent focal adhesions (Nobes and Hall, 1995). In migrating fibroblasts, focal complexes first form at the leading edge of lamellipodia and filopodia induced by Racl activity (Rottner et al., 1999). Subsequently, in a process that requires RhoA activity and actomyosin contraction (Chrzanowska-Wodnicka and Burridge, 1996), focal complexes mature into larger focal adhesions as additional proteins are recruited, linking the adhesion site to the actin cytoskeleton.

\footnotetext{
Received 0ct. 3, 2005; revised Dec. 15, 2005; accepted Dec. 18, 2005.

This work was supported by National Institutes of Health Grant NS41564 and National Science Foundation Grant IBN-0419926 to T.M.G. We thank Kate Kalil and members of the Gomez laboratory for comments on this manuscript and Estuardo Robles for technical support.

Correspondence should be addressed to Dr. Timothy M. Gomez, University of Wisconsin, 257 Bardeen Labs, 1300 University Avenue, Madison, WI 53706. E-mail: tmgomez@wisc.edu.

DOI:10.1523/JNEUROSCI.4209-05.2006

Copyright $\odot 2006$ Society for Neuroscience $\quad$ 0270-6474/06/261418-11\$15.00/0
}

In growth cones, integrin-dependent adhesion sites are referred to as point contacts. By immunocytochemistry, point contacts contain many of the same proteins that localize to focal adhesions in non-neuronal cells, including paxillin, vinculin, $\beta 1$ integrin, and focal adhesion kinase (Renaudin et al., 1999). Interestingly, the Rho GTPases RhoA and RhoB are also enriched at point contacts. Point contacts exhibit different morphologies depending on substrata, suggesting functionally distinct adhesive sites may form in growth cones (Gomez et al., 1996). However, because growth cone point contacts are, on average, 10 times smaller and more punctate in appearance than fibroblastic focal adhesions, it is unclear whether these adhesion sites are regulated in a similar manner. Although it is clear that Rho GTPase activity has profound affects on growth cone motility (Luo, 2000) and guidance (Huber et al., 2003), the role of RhoA or Racl in regulating growth cone-substratum adhesion has not been addressed.

Here, we describe a role for paxillin-containing adhesive point contacts in the stabilization of growth cone lamellipodial and filopodial protrusions. Live imaging of paxillin-green fluorescent protein (GFP)-expressing growth cones shows that punctate point contacts form within the leading edge of protrusions and often expand into larger, more stable adhesions reminiscent of focal contacts. We also show that point contact dynamics depend on coordinated Racl and RhoA activities, which support rapid neurite outgrowth through stabilization of lamellar protrusions. The initial formation of paxillin-containing point contacts at the leading edge of growth cones requires Racl, whereas stabilization of point contacts requires inactivation of Racl and activation of the RhoA target Rho kinase (ROCK). Our results suggest that coordinated Rho GTPase activities promote rapid neurite outgrowth on laminin (LN) through formation of point contacts and stabilization of membrane protrusions. 


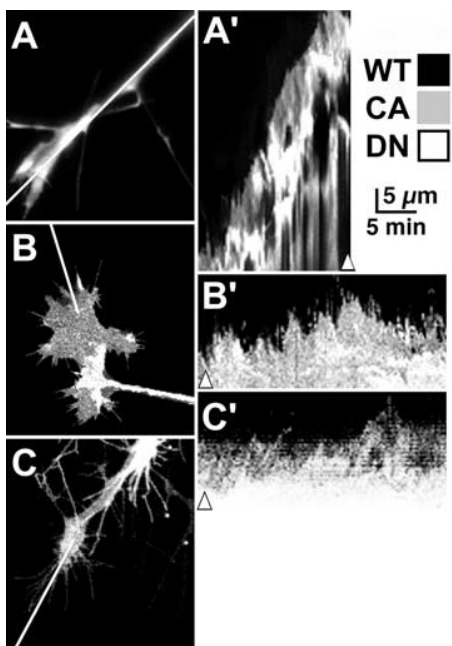

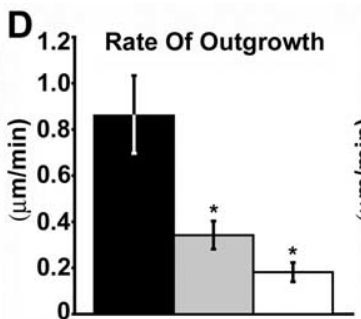

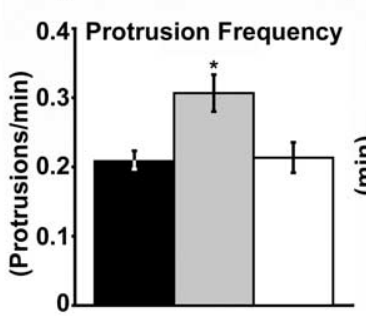

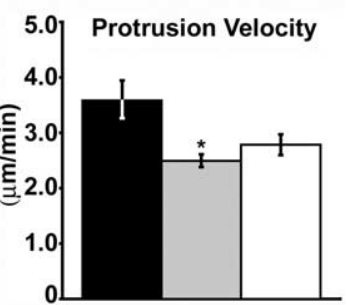

Protrusion

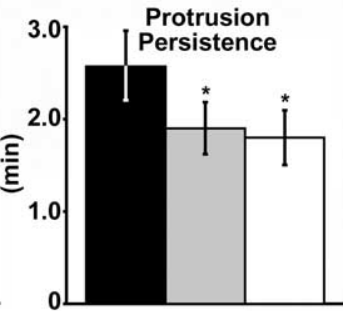

Figure 1. Rac1 mutants reduce neurite outgrowth and alter protrusion dynamics. $A-C$, Representative growth cones expressing GFP $(\boldsymbol{A})$, GFP-CA Rac1 $(\boldsymbol{B})$, or GFP-DN Rac1 $(\boldsymbol{C})$ on $\mathrm{LN} . \boldsymbol{A}^{\prime}-\boldsymbol{C}^{\prime}$, Fluorescent growth cones were imaged every $15 \mathrm{~s}$ for $15 \mathrm{~min}$. Kymographs were generated along the lines indicated in $\boldsymbol{A}-\boldsymbol{C}$. Arrowheads indicate the time points of sampled images in $\boldsymbol{A}-\boldsymbol{C}$. Expanding and contracting contours of the edge indicate lamellar protrusion and withdrawal, respectively, with a sharp slope corresponding to high velocity and a wide base indicating prolonged persistence. $\boldsymbol{D}$, Quantification of the rate of outgrowth as well as protrusion velocity, frequency, and persistence measured from kymographs. Both CA $(n=15)$ and DN $(n=8)$ Rac1 mutants reduced the rate of outgrowth and protrusion persistence compared with control $(n=15)$. CA Rac1 also increased protrusion frequency. ${ }^{*} p<0.05$. Error bars indicate SEM. WT, Wild type.

\section{Materials and Methods}

Embryo injection and cell culture. Expression constructs were subcloned into the Xenopus-preferred PCS + vector (D. Turner, University of Michigan, Ann Arbor, MI). Rac1 mutants were provided by M. Ruchhoeft. cDNA for chicken paxillin was provided by A. F. Horwitz (University of Virginia, Charlottesville, VA). Xenopus laevis embryos were obtained as described previously (Gomez et al., 2003) and staged according to Nieuwkoop and Faber (1994). Two or four blastomeres of eight-cell stage embryos were injected with $100 \mathrm{pg}$ of DNA or $0.5-1 \mathrm{ng}$ of in vitro transcribed, capped mRNA (mMessage Machine; Ambion, Austin, TX). For spinal cord cultures, neural tubes were dissected from stage 22 embryos and explants were cultured in a $1 \times$ modified Ringer's solution. For retinal cultures, eye primordia were dissected from stage 35/36 embryos and explants were cultured in 60\% L15 medium (Sigma, St. Louis, MO) as described previously (Campbell et al., 2001). Explants were plated on glass coverslips coated with $100 \mu \mathrm{g} / \mathrm{ml}$ poly-D-lysine (PDL; Sigma) alone or PDL followed by $10 \mu \mathrm{g} / \mathrm{ml} \mathrm{LN} \mathrm{(Sigma)} \mathrm{as} \mathrm{described} \mathrm{previously} \mathrm{(Go-}$ mez et al., 2003). Cultures were imaged or fixed 12-24 h after plating.

Image acquisition and reagents. For both live and fixed samples, images were acquired using a $60 \times / 1.45$ numerical aperture objective lens on an Olympus (Tokyo, Japan) Fluoview 500 laser-scanning confocal system mounted on an AX-70 upright microscope. Live samples were imaged at $2 \times$ zoom, and fixed samples were imaged at $3 \times$ zoom. Live explant cultures were sealed in enclosed perfusion chambers as described previously (Gomez et al., 2003) to allow rapid exchange of solutions. Y-27632 and ML-7 were purchased from Calbiochem (La Jolla, CA). Recombinant human Sema3A-Fc was purchased from R \& D Systems (Minneapolis, MN). Biotinylated p21-activated kinase-Cdc42/Racl-binding (PAK-CRIB) peptide was synthesized by the University of Wisconsin Peptide Synthesis Facility (Madison, WI) as described previously (Vastrik et al., 1999); internalization was confirmed by FITC-streptavidin staining. The p21-binding domain of PAK1 fused to glutathione-Stransferase (GST-PBD) was provided by W. Bement (University of Wisconsin, Madison, WI). Function-blocking $\beta 1$-integrin antibody was provided by K. Yamada (National Institute of Dental and Craniofacial Research, National Institutes of Health, Bethesda, MD).

Antibodies and labeling. For immunocytochemisty, cultures were fixed in $4 \%$ paraformaldehyde $/ 4 \%$ sucrose in $\mathrm{Ca}^{2+}$ - and $\mathrm{Mg}^{2+}$-free PBS (CMF-PBS), followed by permeabilization in $0.1 \%$ Triton X-100 in CMF-PBS, and blocked in GDB buffer [0.5\% fish gelatin (Sigma) and
0.2\% Triton X-100] in CMF-PBS for $1 \mathrm{~h}$ at room temperature. Primary antibodies were used at the following dilutions: 1:500 paxillin (BD Transduction Laboratories, Franklin Lakes, NJ); 1:000 c-myc (Santa Cruz Biotechnology, Santa Cruz, CA); 1:500 Vinculin (Sigma); 1:500 $\beta 1$-integrin (Developmental Studies Hybridoma Bank, University of Iowa, Iowa City, IA); 1:1000 PY99 (phosphotyrosine; Santa Cruz Biotechnology); 1:1000 GST (Amersham Biosciences, Piscataway, NJ); and 1:250 phospho-myosin light chain 2 (Cell Signaling Technology, Beverly, MA). Alexa-Fluorconjugated secondary antibodies were purchased from Molecular Probes (Eugene, OR) and used at 1:250. A 1:50 dilution of Alexa-546 Phalloidin (Molecular Probes) was used to label F-actin. To detect active Rac1, $5 \mu \mathrm{g} / \mathrm{ml}$ GSTPBD was first applied to growth cones, followed by an anti-GST antibody and fluorescent secondary antibodies, each separately in GBD buffer overnight at $4^{\circ} \mathrm{C}$ as described previously (Li et al., 2002).

Image analysis and quantification. Images were analyzed using MetaMorph software (Universal Imaging, Sunnyvale, CA). Point contacts were identified within the peripheral domain of paxillin-immunofluorescent images as discrete regions of fluorescence that were at least two times greater in intensity than the immediately surrounding cellular background. To quantify the length of individual adhesions, user-defined regions of images were thresholded to highlight areas of discrete fluorescence, and the longest cord was measured from this thresholded area. For time-lapse images, discrete areas containing paxillin-GFP that were at least two times brighter than the surrounding background and remained fixed in place for a minimum of $30 \mathrm{~s}$ were scored as point contacts. Kymographs were created using the kymograph function in MetaMorph along lines one pixel in width. Protrusions were measured if the leading edge membrane exhibited a positive slope with a minimum rise and run of $1.5 \mu \mathrm{m}$ and $30 \mathrm{~s}$, respectively. For display purposes, kymographic images of point contacts were processed with a $3 \times 3$ low-pass kernel in MetaMorph and pseudocolored in Image (W. Rasband, National Institutes of Health). For all other figures, images were processed in Photoshop (Adobe Systems, San Jose, CA) as follows: brightness levels adjusted, unsharp mask routine to improve edge detection, converted to 8-bit depth, and cropped. Statistical significance was determined using Mann-Whitney $t$ test, and variance was reported as \pm SEM using InStat software (GraphPad Software, San Diego, CA).

\section{Results}

Balanced Racl activity promotes growth cone motility by stabilizing membrane protrusions

To assess the function of Rac1 in motile growth cones, we expressed dominant-negative (DN) or constitutively active (CA) Racl mutants as GFP fusion proteins in Xenopus spinal neurons. The DN N17 Rac1 mutant produces a nonfunctional protein that sequesters upstream exchange factors, whereas V12 Rac1 is CA because of its inability to hydrolyze GTP. Consistent with previous reports (Kuhn et al., 1998; Ruchhoeft et al., 1999), activating or inhibiting Rac1 in neurons altered growth cone morphologies and significantly reduced the rate of axon outgrowth (Fig. 1). Typically, growth cones expressing CA Racl had an increased lamellipodial area, whereas growth cones expressing DN Rac1 had little or no veil and several long, stable filopodia.

The effects on growth cone morphology likely result from altered protrusive behavior of the leading edge, which we quantified by kymography. Kymography allows us to measure several 
characteristics of leading edge protrusions, including the frequency, rate, and persistence of protrusions (Hinz et al., 1999) (see Materials and Methods). To generate kymographs, userdefined lines spanning the leading edge of GFP-fluorescent growth cones are sampled at each frame of a time-lapse sequence and montaged side by side into a single image. Therefore, each kymograph represents the protrusive behavior of an individual growth cone over time. Kymographs revealed that the leading edge of wild-type growth cones steadily advanced forward over a 30 min observation period, whereas there was little to no net forward advance of the leading edge of either DN Rac1- or CA Rac1-expressing growth cones (Fig. $1 A^{\prime}-C^{\prime}$ ). However, expression of Racl mutants did not consistently nor significantly reduce the velocity or the frequency of individual membrane protrusions (Fig. 1D). On the contrary, CA Racl increased the frequency of veil protrusions significantly above control growth cones (Fig. $1 D)$, consistent with the role of Racl in promoting actin polymerization. On the other hand, the persistence of protrusions was dramatically and consistently reduced in both DN and CA Rac1expressing growth cones compared with control (Fig. 1D). Together, these results suggest that balanced Racl activity promotes axon outgrowth by stabilizing growth cone membrane protrusions.

\section{Perturbing Rac1 activity alters point contact adhesions}

Inhibiting or overactivating Racl reduced the persistence of lamellipodial protrusions, suggesting that cell-substratum adhesion is disrupted. To test whether adhesion complexes are altered under these conditions, we immunocytochemically labeled growth cones expressing Racl mutants with an antibody to paxillin, an adaptor protein that clusters at sites of adhesion (Geiger et al., 2001) (Fig. 2). Normally, paxillin localizes to discrete puncta within lamellipodia and filopodia of growth cones; these paxillin-rich structures varied in shape and size, ranging from small foci to oblong streaks resembling point contacts and small focal adhesions, respectively (Fig. 2A). Wild-type growth cones maintained an average of $8.76 \pm 0.89(n=46)$ paxillincontaining point contacts with an average size of $0.78 \pm 0.03 \mu \mathrm{m}$ (longest cord; see Materials and Methods). The number of point contacts formed by CA Rac1-expressing growth cones increased but was not significantly greater than control (Fig. 2 D). However, CA Racl strongly reduced the average size of point contacts (Fig. $2 E)$. In contrast, growth cones expressing DN Rac1 had consistently fewer point contacts per growth cone, but the average size of contacts was similar to control (Fig. $2 D, E$ ). These results suggest that although Racl activity may be required for point contact formation, the stabilization and maturation of point contacts may require reduced Racl activity.

\section{Live imaging of paxillin-GFP}

Immunocytochemical experiments reveal the static distribution of paxillin in fixed cells but cannot address whether altering Rac1 activity affects the assembly or disassembly of these adhesion sites. To study the dynamic assembly and turnover of point contacts, we used time-lapse imaging of live growth cones expressing paxillin fused to GFP (movie 1, available at www.jneurosci.org as supplemental material). Expressing paxillin-GFP did not affect the average rate of neurite outgrowth (paxillin-GFP, $1.01 \pm 0.16$ $\mu \mathrm{m} / \mathrm{min}, n=17$, versus GFP only, $0.86 \pm 0.17 \mu \mathrm{m} / \mathrm{min}, n=22$; $p=0.33$ ). To confirm the proper localization of this fusion protein, we immunofluorescently labeled paxillin-GFP-expressing growth cones for other adhesion site markers. We found that paxillin-GFP colocalizes with several markers of focal adhesions,
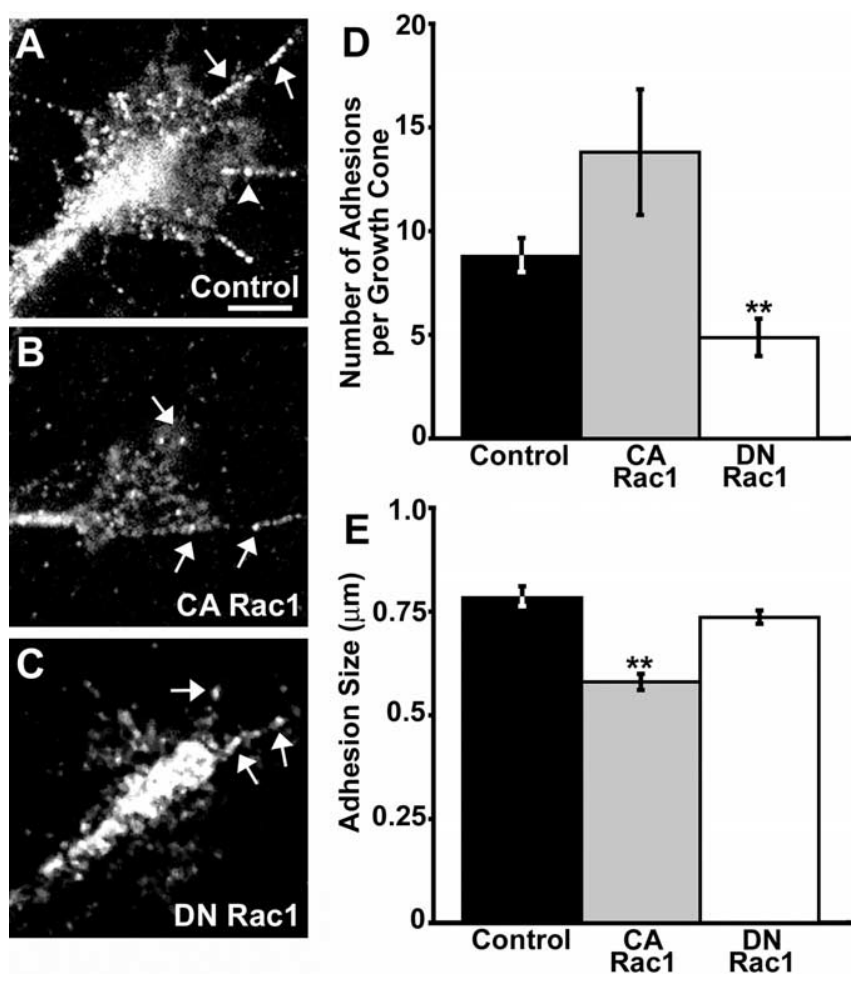

Figure 2. Perturbing Rac1 activity alters paxillin-containing point contacts. $\boldsymbol{A}-\boldsymbol{C}$, Representative control, CA, or DN Rac1-expressing growth cones on LN stained with an antibody against paxillin. Note that control growth cones $(n=46)$ have both elongated, streak-like (arrows) as well as smaller, punctate (arrowhead) point contacts. Expression of CA Rac1 $(n=10)$ results in smaller and more punctate point contacts $(\boldsymbol{B})$, whereas DN Rac1-expressing growth cones $(n=$ 37) have fewer point contacts overall (C). D, E, Quantification of point contact size (D) and number of point contacts per growth cone $(\boldsymbol{E})$. Point contact size was determined by measuring the length along the longest cord (see Materials and Methods). Scale bar, $5 \mu \mathrm{m}$. ${ }^{* *} p<0.001$. Error bars indicate SEM.

including vinculin, $\beta 1$-integrin, and phosphotyrosine (Fig. 3). Similar to endogenous protein, paxillin-GFP organized into discrete puncta and streak-like structures within filopodia and peripheral lamellipodia (Fig. 4). Analysis of paxillin-GFP in live growth cones showed that paxillin-containing adhesions are highly dynamic: assembling at the leading edge, growing in size, and disassembling while remaining in a fixed position as the growth cone migrates forward (Fig. $4 A$ ).

Kymographs generated from growth cone regions containing assembling adhesion sites showed both temporal ( $x$-axis) and spatial ( $y$-axis) point contact kinetics (Fig. $4 B, C$ ). Point contacts elongate rearward (toward the growth cone central domain), gradually increasing in both length and fluorescent intensity over an average lifetime of $2.26 \pm 0.18 \mathrm{~min}$. Disassembly, in contrast, occurs rapidly and with no consistent directional bias. Together, these observations suggest that paxillin-GFP is a reliable marker to track adhesion site dynamics in live growth cones.

\section{LN promotes Rac1-dependent point contact assembly}

To study de novo point contact assembly, we examined the redistribution of paxillin-GFP during acute stimulation with LN. For this assay, paxillin-GFP-expressing growth cones were plated on PDL, a non-integrin-binding substratum that does not support point contact formation (Fig. 5). Growth cones were imaged $5-10 \mathrm{~min}$ before and $10 \mathrm{~min}$ after the addition of $25 \mu \mathrm{g} / \mathrm{ml} \mathrm{solu}-$ ble LN. On PDL, paxillin-GFP was distributed in a diffuse pattern that paralleled cell volume similar to unconjugated GFP (Fig. 

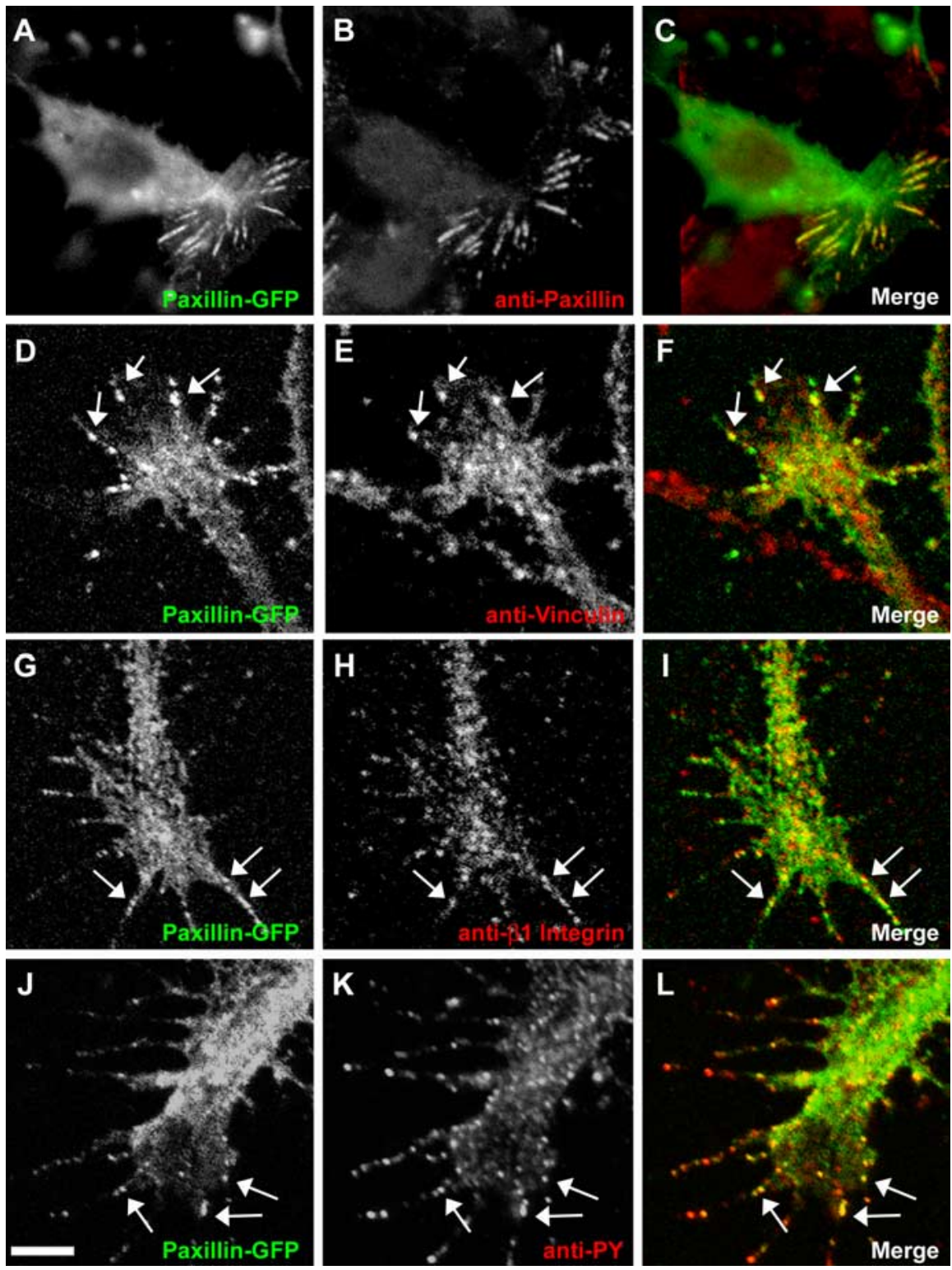

Figure 3. Paxillin-GFP colocalizes with adhesion markers. A-C, Paxillin-GFP in a non-neuronal cell colocalizes with antipaxillin antibody. Similar streak-like distributions are observed in immunolabeled non-neuronal cells not expressing paxillinGFP. $\boldsymbol{D}-\boldsymbol{L}$, Growth cones expressing paxillin-GFP were stained with antibodies against vinculin $(\boldsymbol{D}-\boldsymbol{F}), \beta 1$-integrin $(\mathbf{G}-\boldsymbol{I})$, and phosphotyrosine (PY) $(\boldsymbol{J}-\boldsymbol{L})$. Arrows indicate areas of colocalization within point contacts. Scale bar, $5 \mu \mathrm{m}$.

$5 A)(n=15)$. However, after an average delay of $6.35 \pm 0.62 \mathrm{~min}$, the addition of soluble LN promoted paxillin-GFP clustering into discrete puncta and streak-like structures resembling point contacts within leading edge protrusions. This occurred in $80 \%$ of the growth cones observed (Figs. 5A, 6D) (movie 2, available at www.jneurosci.org as supplemental material).

Analysis of the morphological changes induced by acute LN stimulation showed biphasic effects on membrane protrusion and the rate of axon outgrowth (Fig. $5 B, C$ ). Immediately after $\mathrm{LN}$ addition, growth cones exhibited a slight increase in the duration of protrusions as well as a modest increase in their rate of outgrowth. However, after $6 \mathrm{~min}$ of LN addition and concurrent with the appearance of point contacts, there was a much more robust increase in protrusion persistence and accelerated axon outgrowth (Fig. $5 B, C$ ). The assembly of point contacts and increased persistence of protrusions correlated closely with LN coating to the substratum (Fig. 5E). Therefore, the biphasic ef- fects of LN stimulation may be attributable to the growth cones responding initially to soluble LN with increased protrusion and to bound LN with increased point contact formation. These results are consistent with the notion that rapid axon outgrowth requires stabilization of protrusions by paxillin-containing point contacts.

The molecular basis for the effects of acute LN stimulation on growth cone motility and point contact formation likely involves integrin activation of Rho GTPase signaling (Kuhn et al., 1998). To test whether the effects of LN stimulation were integrin mediated, we inhibited $\beta 1$ integrin receptors with a functionblocking antibody (Akiyama et al., 1989) (Fig. 6A). Growth cones expressing paxillin-GFP were pretreated with $100 \mu \mathrm{g} / \mathrm{ml}$ $\beta 1$-integrin antibody for $20-30 \mathrm{~min}$ before the addition of $25 \mu \mathrm{g} / \mathrm{ml} \mathrm{LN}$. Surprisingly, the function-blocking $\beta 1$-integrin antibody alone slightly increased the rate of outgrowth, possibly because of receptor clustering (Akiyama et al., 1989). Moreover, the addition of the functionblocking antibody alone also induced small paxillin-GFP puncta in $45.4 \%$ of the growth cones observed (Fig. $6 D)(n=11)$. However, antibody-induced puncta appeared smaller and were more transient than structures we would normally define as point contacts (data not shown). Importantly, the addition of LN in the continued presence of function-blocking antibodies did not increase the number or the morphology of these puncta or promote the formation of bona fide point contacts (Fig. 6A,D). Moreover, stimulation by LN did not significantly further increase neurite outgrowth in the presence of functionblocking antibodies (Fig. 6 E). For remaining growth cones that did not respond to $\beta 1$-integrin antibody alone, paxillin-GFP remained diffusely distributed before and up to 10 min after the addition of LN plus antibody. Therefore, stimulation of axon outgrowth and point contact formation by $\mathrm{LN}$ requires $\beta 1$-integrin activation.

To determine whether Racl is required for the $\mathrm{LN}$-induced assembly of point contacts downstream of integrins, we blocked Racl activity using two approaches. First, we coexpressed myctagged DN Racl together with paxillin-GFP (Fig. 6B). Expression of DN Racl blocked LN-induced point contact formation and growth cone motility in all growth cones tested $(n=9)$ (Fig. $6 D, E)$. We also acutely inhibited Racl with a cell-permeable peptide inhibitor consisting of the CRIB domain of PAK1 fused to the Antennepedia internalization sequence (Vastrik et al., 1999) (Fig. $6 C)$. Growth cones expressing paxillin-GFP were treated with 60 $\mu \mathrm{g} / \mathrm{ml}$ PAK-CRIB for $5 \mathrm{~min}$ before the addition of LN. Only $18.2 \%$ of the treated growth cones $(n=11)$ assembled point contacts in response to LN (Fig. 6D), and there was no significant increase in the rate of axon outgrowth (Fig. 6 E). Although lamellipodial extensions are compromised with Racl inhibited, 

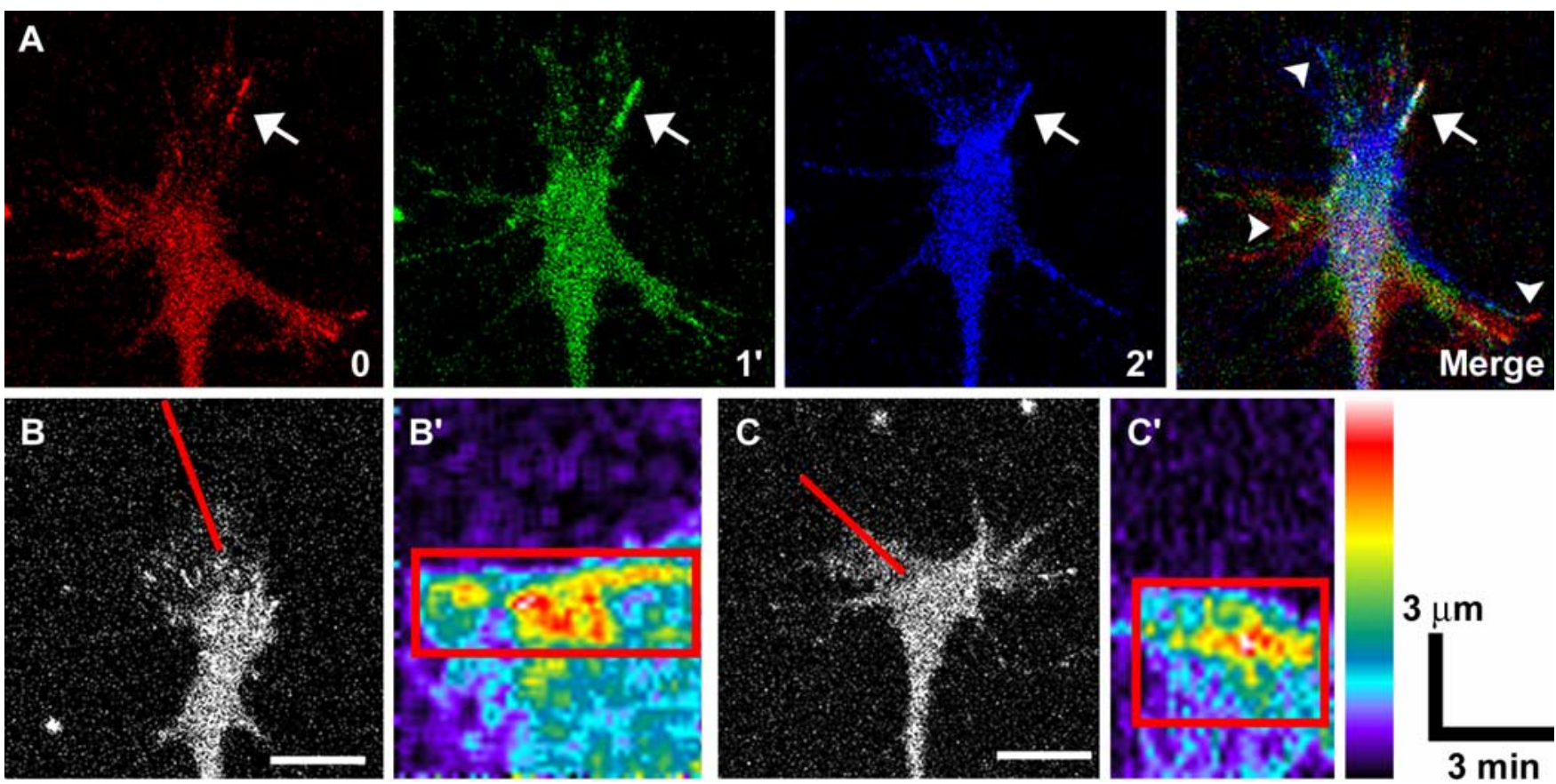

Figure 4. Live imaging of paxillin-GFP in growth cones. Paxillin-GFP is organized into point contacts that dynamically assemble, grow, and disassemble during growth cone advance on LN. $\boldsymbol{A}$, Pseudocoloring three time points separated by 1 min red, green, and blue help identify stable regions that display as white in the merged image. Large, stable point contacts appear white in the merged image (arrow), whereas more transient contacts appear as separate colors (arrowheads). See supplemental material (available at www.jneurosci.org) for the movie corresponding to this growth cone (movie 1). $\boldsymbol{B}, \boldsymbol{C}$, Kymographs through individual point contacts $\left(\boldsymbol{B}^{\prime}, \boldsymbol{C}^{\prime}\right)$ assembled from the lines indicated in the frames to the left show the dynamic assembly of individual point contacts. The pseudocolor scale indicates bright fluorescence as hot colors. Note that point contacts tend to elongate proximally and that fluorescence intensity increases with elongation (red boxes). Scale bars, $5 \mu \mathrm{m}$. The calibration bars refer to the kymographs.

growth cones can extend protrusions under these conditions (Fig. 1C,D), suggesting that reduced point contact formation is not simply attributable to reduced protrusiveness.

Engagement of integrin receptors has been shown to activate Racl in non-neuronal cells (Del Pozo et al., 2002). To determine whether Racl activity is elevated by LN stimulation in growth cones, we used an in situ binding assay shown previously to detect activated Rho GTPases in growth cones (Li et al., 2002). Briefly, neurons were fixed $10 \mathrm{~min}$ after $\mathrm{LN}$ addition and probed for active Rac1 using GST-PBD. Although this region of PAK1 can bind both Racl and Cdc42 (Bokoch, 2003), Cdc42 does not appear to bind to GST-PBD in Xenopus neurons (Li et al., 2002), suggesting this probe is specific for active Racl in our system. Racl activity, as assessed by the fluorescence intensity of individual growth cones, was significantly higher in LN-stimulated growth cones compared with control (Fig. 7). However, when growth cones were treated with the $\beta 1$-integrin functionblocking antibody before LN stimulation, the average fluorescence intensity did not significantly differ from control growth cones on PDL alone. Together, these results suggest that LN supports rapid axon outgrowth by activating Racl downstream of $\beta 1$-integrin receptors, leading to the formation of paxillincontaining point contacts and stabilization of membrane protrusions.

\section{Racl inactivation is required for point contact stabilization}

Our findings suggest that Racl is required for the initial assembly of point contacts. However, overactivation of Racl produced many small but few large point contacts (Fig. 2), suggesting that stabilization and maturation of adhesion sites may depend on reduced Racl activity. To determine whether Racl plays a role in point contact stability, we compared paxillin-GFP dynamics of wild-type and CA Rac1-expressing growth cones on LN. Wildtype growth cones assemble both stable and transient paxillincontaining point contacts (see movie 1, available at www.jneurosci.org as supplemental material). A significant percentage of point contacts in control growth cones were highly unstable with lifetimes of $<1 \mathrm{~min}$ (Fig. $8 \mathrm{D}$ ). These adhesions do not elongate, but remain as symmetrical puncta. However, some point contacts in wild-type growth cones are stabilized with lifetimes between 3 and $8 \mathrm{~min}$. Stable point contacts often began as small puncta that elongated over time into streak-like structures, reaching maximal lengths of $>1 \mu \mathrm{m}$ (Fig. $8 A, B$ ). In contrast, growth cones coexpressing CA Racl and paxillin-GFP produced fewer stable point contacts and proportionately more transient point contacts (Fig. 8C,D) (movie 3, available at www.jneurosci.org as supplemental material). Thus, overactivation of Rac1 seems to interfere with stabilization and elongation of point contacts.

\section{ROCK inhibition alters leading edge protrusion and point contact dynamics similar to CA Rac1}

The Rho GTPases Racl and RhoA are thought to have antagonistic roles during axon pathfinding (Luo, 2000). However, although strong activation of RhoA is known to induce growth cone collapse and neurite retraction (Jalink et al., 1994), a low level of local RhoA activity may be necessary for growth cone migration (Nakamura et al., 2005), possibly through the stabilization of adhesion sites (Ridley et al., 2003). The RhoA effector ROCK is necessary for focal adhesion formation in fibroblasts (Chrzanowska-Wodnicka and Burridge, 1996), but a similar function of ROCK in growth cones has not been demonstrated. ROCK promotes myosin-II contraction by both directly phosphorylating the activating serine residue of myosin light chain 


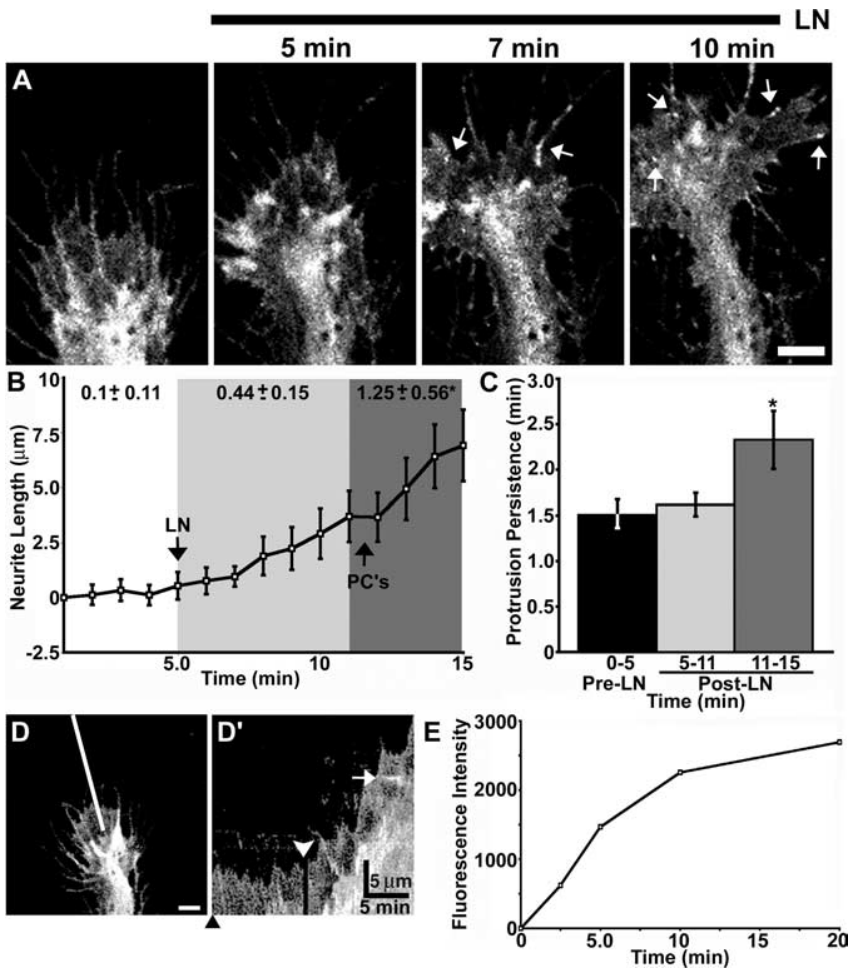

Figure 5. Laminin promotes point contact assembly and increased neurite outgrowth. $\boldsymbol{A}$ Growth cone expressing paxillin-GFP on PDL before and at the indicated times after the addition of $25 \mu \mathrm{g} / \mathrm{ml} \mathrm{LN}$. Within $10 \mathrm{~min}$ of LN addition, several point contacts are obvious (arrows). The mean time to the first identifiable point contact is $6.35 \pm 0.62 \mathrm{~min}(n=12)$. See supplemental material (available at www.jneurosci.org) for the movie corresponding to this growth cone (movie 2). $\boldsymbol{B}$, Time-lapse quantification of neurite outgrowth $(n=15)$ reveals a biphasic increase in the rate of outgrowth induced by LN (at $5 \mathrm{~min}$ ). The rate of outgrowth increases slightly immediately after $L N$ addition (light gray region). However, by 7 min after stimulation with LN (dark gray region), the rate of outgrowth increases robustly, which corresponds near the time when the first point contacts ( $P C^{\prime}$ 's) form. C, Quantification of protrusion persistence shows that point contact formation correlates with a significant increase in protrusion stability (colored regions match in $\boldsymbol{B}$ and $\boldsymbol{C}$ ). $\boldsymbol{D}, \boldsymbol{D}^{\prime}$, Kymography qualitatively illustrates the changes in outgrowth and protrusion induced by LN. The kymograph in $\boldsymbol{D}^{\prime}$ assembled along the line indicated in $\boldsymbol{D}$. The black arrowhead indicates the time point of sampled image in $\boldsymbol{D}$. The white arrowhead indicates the time of $L N$ addition. Note that the point contact was captured by this kymograph (white arrow). $\boldsymbol{E}$, Quantification of LN-coating dynamics. LN was added to glass coverslips pretreated with PDL, fixed at various time points, and stained with an anti-LN antibody. Fluorescence intensity of the LN immnunostaining over time shows that LN binds the substratum rapidly and in a saturatable manner with a time course that is consistent with the appearance of point contacts, increased protrusion persistence, and accelerated neurite outgrowth. Scale bars, $5 \mu \mathrm{m}$. Calibration bars are as indicated. ${ }^{*} p<0.05$. Error bars indicate SEM.

(Amano et al., 1996), and phosphorylating and inhibiting myosin light chain phosphatase (Kimura et al., 1996). Because Rac1 is known to antagonize RhoA signaling (Rottner et al., 1999), we tested whether the effects we observed on point contact dynamics induced by CA Racl could be mimicked by direct inhibition of ROCK activity.

Using kymography, we compared the persistence, height, velocity, and frequency of protrusions before and after pharmacological inhibition of ROCK with Y-27632 (Calbiochem) (Fig. 9). Similar to our observations with CA Rac1, we found that $25 \mu \mathrm{M}$ Y-27632 significantly decreased protrusion persistence (Fig. 9C). This is qualitatively clear from the kymographs, which appear more irregular in ROCK-inhibited growth cones, indicating unstable protrusions (Fig. $9 A^{\prime}, B^{\prime}$ ).

We also examined the effects of ROCK inhibition on the size and assembly dynamics of growth cone point contacts. First, growth cones treated with Y-27632 were fixed and stained with an antibody against paxillin, and the lengths of paxillin-containing point contacts were measured. Similar to CA Racl expression, treatment with $25 \mu \mathrm{M}$ Y-27632 for 30-45 min significantly reduced the size of point contacts compared with control (Fig. 9D). Next, the effects of ROCK inhibition on point contact dynamics were assessed in live growth cones expressing paxillin-GFP. Again, similar to CA Rac1, Y-27632 significantly decreased the percentage of stable point contacts while increasing the percentage of labile point contacts (Fig. 9E). These results suggest that modest or localized RhoA activity may be necessary to locally activate ROCK, which stabilizes growth cone point contacts to promote persistent membrane protrusions.

CA Racl and inhibition of ROCK reduce myosin-II activity Downstream Racl signaling is known to reduce myosin-II-based contraction (Sanders et al., 1999; van Leeuwen et al., 1999), whereas the RhoA/ROCK pathway increases myosin activity (Amano et al., 1996; Kimura et al., 1996). To determine whether decreased myosin-II activity correlates with the loss of stable point contacts in growth cones, we assayed phospho-myosin light chain (p-MLC) levels in CA Rac1-expressing and Y-27632treated growth cones. Using a phospho-specific antibody, we measured the average fluorescence intensity of p-MLC immunolabeling in control and experimental growth cones (Fig. 9F-H). We found that both expression of CA Racl and inhibition of ROCK significantly decreased MLC phosphorylation relative to controls (Fig. 9J). As a positive control for the p-MLC antibody, we demonstrated that pharmacological inhibition of myosin light chain kinase with ML-7 (Calbiochem) also significantly decreased p-MLC staining in growth cones.

\section{Semaphorin 3A disrupts point contact formation and stabilization}

Many axon guidance cues have been shown to signal through the Rho GTPases (Huber et al., 2003), but the effects of axon guidance cues on point contact dynamics has not been examined. Semaphorin 3A (Sema3A) is a secreted repulsive guidance cue that activates Rac1 through the neuropilin-1/Plexin A1 receptor complex (Turner et al., 2004) and more recently has been shown to promote local synthesis of RhoA mRNA (Wu et al., 2005). Interestingly, Sema3A also reduces integrin-dependent adhesion of both neuronal growth cones and vascular endothelial cells (Mikule et al., 2002; Serini et al., 2003), but the underlying mechanism remains unclear. Because we have shown that Rac1 regulates point contact dynamics in an integrin-dependent manner, we tested whether the addition of Sema3A alters the assembly or stabilization of paxillin-containing point contacts in growth cones.

Although Xenopus spinal neurons are repelled away from a local source of Sema3A, these neurons do not appear to respond to globally applied Sema3A (Song et al., 1998) (Fig. S1, available at www.jneurosci.org as supplemental material). Therefore, to test the effects of bath-applied Sema3A on point contacts, we switched to Xenopus retinal ganglion cell (RGC) axons, because Sema3A collapses these axons in a dose-dependent manner (Campbell et al., 2001) (Fig. S1, available at www.jneurosci.org as supplemental material). However, because we cannot measure paxillin-GFP puncta within fully collapsed growth cones, we tested the effects of a low, noncollapsing dose of recombinant Sema3A (400 ng/ml; R \& D Systems). At this concentration, the rate of RGC neurite outgrowth on LN is modestly reduced $(0.67 \pm 0.16 \mu \mathrm{m} / \mathrm{min})$ in the presence of Sema3A compared with 
the pretreatment rate of outgrowth $(0.9 \pm$ $0.14 \mu \mathrm{m} / \mathrm{min} ; n=15 ; p=0.3)$. Sema3A also had subtle effects on protrusion dynamics, although the persistence, distance, and velocity of membrane protrusions were not significantly altered at this concentration (Fig. S2, available at www. jneurosci.org as supplemental material).

Despite the modest effects on growth cone motility of this subcollapsing dose, the addition of $400 \mathrm{ng} / \mathrm{ml}$ Sema3A rapidly altered paxillin-GFP dynamics. Within minutes of Sema3A addition, most of the pre-existing paxillin-containing point contacts were disassembled (Fig. 10 A) (see movie 4, available at www.jneurosci.org as supplemental material). Moreover, new point contacts formed at a much lower frequency than in control growth cones (Fig. $10 B)$. Consistent with Sema3A-activating Rac1, new point contacts were more transient and fewer stable point contacts were formed compared with control (Fig. 10C). These results suggest that the repulsive cue Sema3A alters the assembly and turnover of adhesion sites, which is consistent with previous reports showing that Sema3A reduces integrin-dependent adhesion. It is noteworthy that this low concentration of Sema3A was able to affect point contact formation and lifetime but did not significantly change membrane protrusion. The apparent disconnect between point contact and protrusion dynamics observed here may be attributable to additional signals activated by Sema3A, such as increased RhoA expression (Wu et al., 2005).

\section{Discussion}

Growth cone adhesion to the ECM is mediated by point contacts that are enriched in adhesion molecules such as $\beta 1$-integrin, paxillin, vinculin, and zyxin (Gomez et al., 1996; Renaudin et al., 1999), but the mechanisms regulating point contact dynamics in growth cones are unknown. In this study, we define a Rho GTPase signaling cascade that regulates point contact assembly and stability. Racl promotes the initial formation of small, labile point contacts downstream of activated integrin receptors. However, active Racl appears to inhibit further stabilization of point contacts. Instead, point contact stabilization depends on deactivation of Racl and activation of the RhoA/ROCK pathway. Pharmacological inhibition of ROCK reduced the percentage of stable point contacts, similar to expression of CA Rac1. Furthermore, both activation of Racl and inhibition of ROCK decreased levels of active myosin-II, implicating actomyosin contraction in growth cone point contact stabilization. Finally, our data suggest that the regulation of point contact dynamics is an important determinant of axon guidance as acute stimulation with LN promotes point contact assembly and axon outgrowth, whereas acute treatment with a low dose of Sema3A disrupts point contacts and slows axon outgrowth.
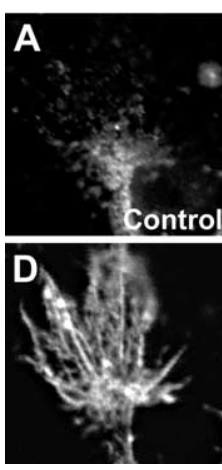

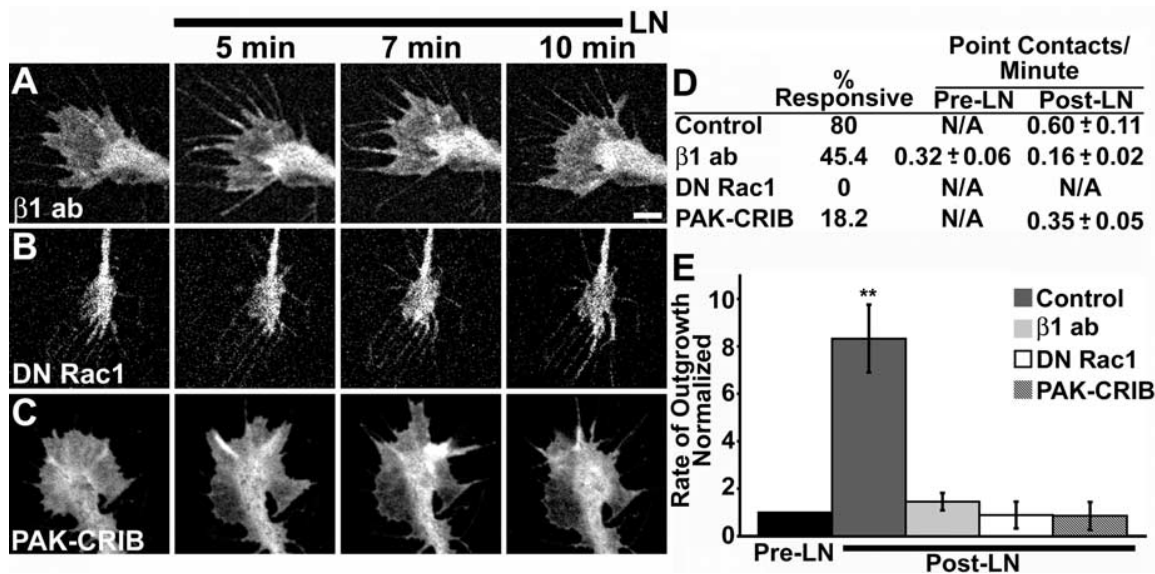

Figure 6. LN-induced point contact assembly and increased outgrowth requires $\beta 1$-integrin and Rac1. $A-C$, Growth cones expressing paxillin-GFP on PDL before and at the indicated times after the addition of $25 \mu \mathrm{g} / \mathrm{ml} \mathrm{LN}$. Unlike control growth cones, antibody $(\boldsymbol{A} ; n=11)$, coexpress DN Rac1 $(\boldsymbol{B} ; n=9)$, or are treated with $60 \mu \mathrm{g} / \mathrm{ml}$ PAK-CRIB peptide $(\boldsymbol{C} ; n=11)$. D, Quantifi作 Of those that had identifiable point contacts, the frequency at which they appear (number per minute) was

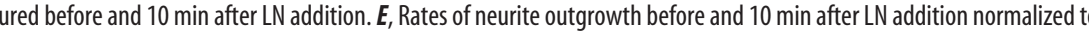
follows: raw intensity values for each growth cone in all conditions were normalized against the average control intensity value.
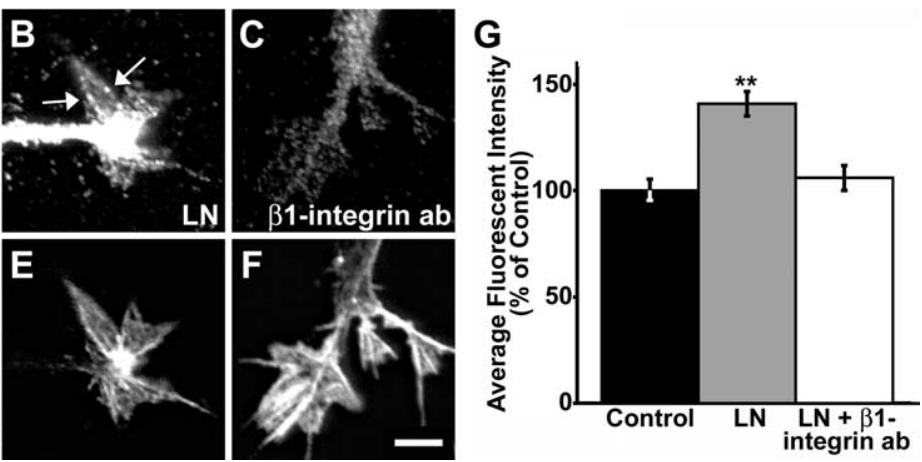

Figure 7. Laminin increases Rac1 activity. $\boldsymbol{A}-\boldsymbol{F}$, Growth cones colabeled for active Rac1 using GST-PBD ( $\boldsymbol{A}-\boldsymbol{C}$; see Materials and Methods) and filamentous actin using fluorescent phalloidin (D-F). Growth cones that were stimulated with $25 \mu \mathrm{g} / \mathrm{ml} \mathrm{LN}$ for 10 min before fixation $(\boldsymbol{B})$ appear brighter than unstimulated controls $(\boldsymbol{A})$, indicating increased Rac1 activity. Arrows point to antibody $(\boldsymbol{C})$ appear similar to unstimulated control growth cones. $\boldsymbol{G}$, Quantification of GST-PBD binding. The average fluorescence intensity was measured within the entire growth cone using MetaMorph software. The addition of $\mathrm{LN}(n=34)$ significantly elevated Rac1 activity compared with control $(n=40)$; this increase was blocked by pretreatment with $\beta 1$-integrin functionblocking antibody $(n=38)$. Scale bar, $5 \mu \mathrm{m} .{ }^{* *} p<0.001$. Error bars indicate SEM. ab, Antibody.

Although many studies have demonstrated the importance of Rac1 for axon outgrowth and guidance (Kuhn et al., 1998; Jurney et al., 2002; Ng et al., 2002; Shekerabi and Kennedy, 2002), these studies have focused on the role of Rac1 in promoting actin polymerization during leading edge protrusion and endocytosis. Here, we show that Racl also supports axon outgrowth by promoting point contact formation, which leads to stabilization of protrusions. However, if inhibiting Racl reduces the number or size of protrusions, then the loss of point contacts in growth cones expressing DN Racl or treated with the PAK-CRIB peptide inhibitor may be a secondary effect of diminished protrusiveness. However, two findings argue against this possibility. First, growth cones can make lamellar protrusions even when Racl signaling is comprised (Fig. 1). Second, growth cones are protrusive on PDL but do not assemble point contacts (Fig. 5), suggesting protru- 

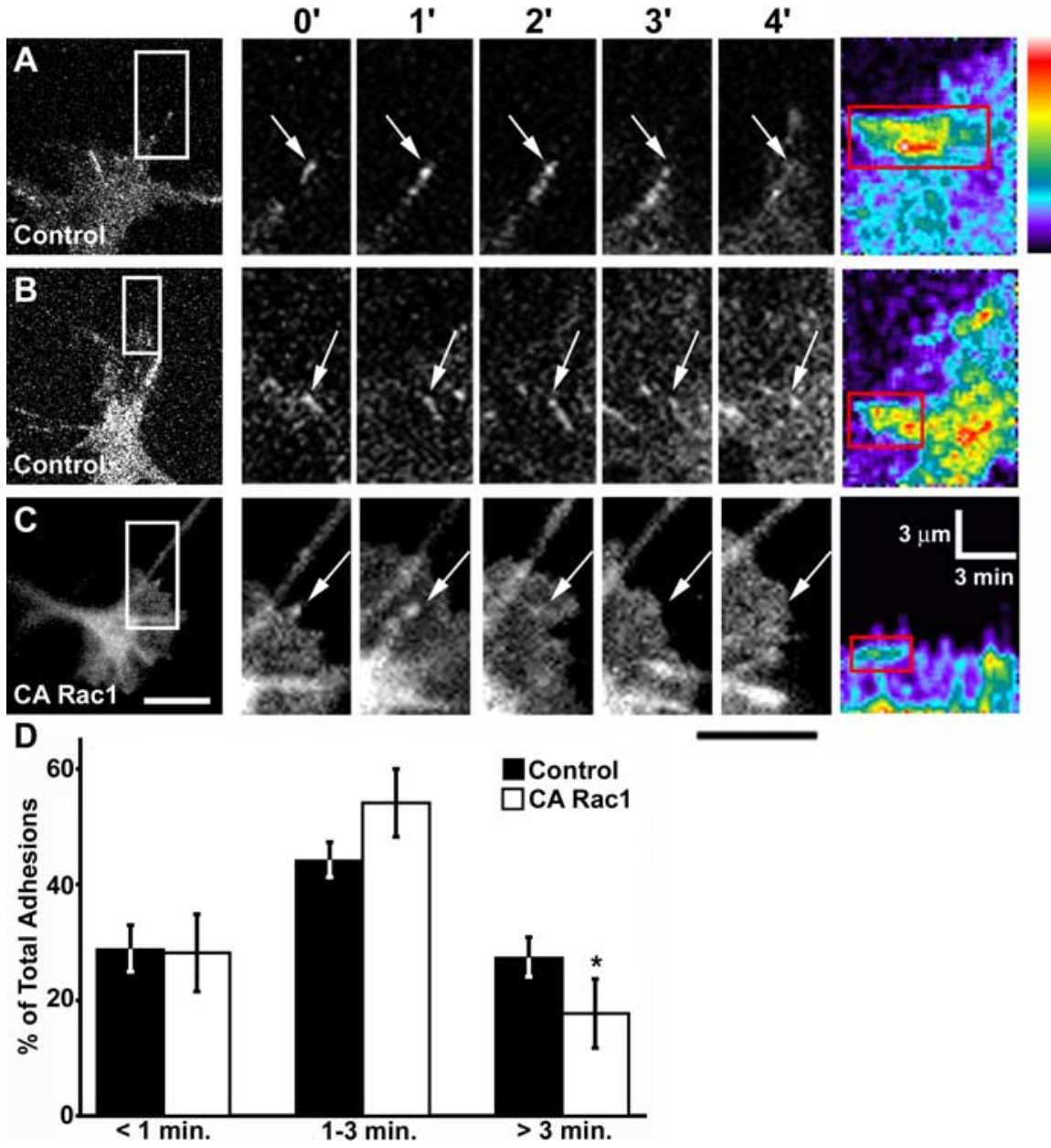

Control 口CA Rac1

Figure 8. CA Rac1 reduces the percentage of stable point contacts. $A-C$, The first panel in each row shows representative growth cones on $\mathrm{LN}$ expressing either paxillin-GFP alone $(\boldsymbol{A}, \boldsymbol{B})$ or with myc-CA Rac1 (C). Subsequent panels magnify the boxed regions at 1 min intervals. The arrows indicate point contacts. In control growth cones, point contacts can become stabilized for several minutes and elongate into streak-like structures resembling focal adhesions. Coexpression of CA Rac $1(\boldsymbol{C})$ results in more transient point contacts that do not elongate. See supplemental material (available at www.jneurosci.org) for the movie corresponding to this growth cone (movie 7). Pseudocolor kymographs at the end of each row show that CA Rac1 decreases the length, intensity, and lifetime of point contacts (red boxes). The pseudocolor scale indicates bright fluorescence as hot colors. D, Distribution of point contact lifetimes. CA Rac1 $(n=16)$ significantly reduces the percentage of stable ( $>3 \mathrm{~min})$ adhesion sites compared with control $(n=16)$. Scale bars, $5 \mu \mathrm{m}$. The calibration bars are as indicated. ${ }^{*} p<0.05$. Error bars indicate SEM.

affected, suggesting that protrusion instability accounted for the reduction in growth cone advance. Here, we show that CA Rac1 expression reduces protrusion stability, while promoting the generation of small, highly labile paxillin-containing point contacts. In control growth cones, these small point contacts often appear to be precursors of larger and more stable adhesion sites. Therefore, persistent growth cone advance may require the maturation of initial point contacts into larger adhesion sites to stabilize leading edge protrusions.

After point contact assembly begins, our results suggest that Racl activity must be locally reduced to allow RhoAdependent signals to promote further elongation and stabilization of point contacts. In general, RhoA/ROCK signaling and actomyosin contraction are considered inhibitors of growth cone advance because RhoA/ROCK are activated downstream of repulsive guidance cues (Shamah et al., 2001; Niederost et al., 2002; Swiercz et al., 2002), leading to growth cone collapse (Jalink et al., 1994). However, our results suggest that a modest level of ROCK activity and myosin-II-based contraction is necessary for the formation of stable, fully elongated point contacts. These structures most likely provide anchorage to the substratum that supports additional contractile forces generated during growth cone advance. This mechanism is consistent with the "substrate-cytoskeletal coupling" model of growth cone advance (Suter and Forscher, 2000; Mitchison and Kirschner, 1988), which states that cell-surface receptors linked to the actomyosin machinery bound to a noncompliant substratum will generate enough tension to pull the growth cone

siveness is not sufficient for point contact formation. These findings support a specific role for Racl in point contact formation and protrusion stabilization.

$\mathrm{Racl}$ is believed to function as a positive regulator of neurite outgrowth downstream of growth-promoting axon guidance cues because of its ability to stimulate actin polymerization and lamellar extension (Luo, 2000). Consistent with this notion, we found that expression of CA Racl enhanced protrusion frequency. However, despite more frequent protrusions, the rate of neurite outgrowth was less than wild type, suggesting that membrane protrusion alone is not sufficient to promote outgrowth. We found that reduced rate of outgrowth was most likely attributable to decreased persistence or stability of protrusions. Previous work in PC12 cells concluded that the stability of protrusions depends on growth cone-substratum adhesion (Varnum-Finney and Reichardt, 1994). In this study, the adhesion-related protein vinculin was knocked down by antisense RNA, which resulted in significantly less stable lamellipodial and filopodial protrusions and attenuated neurite extension. Other parameters measured such as the rates of lamellar extension and retraction were not forward. Without strong linkage to the substratum, actomyosin contraction drives retrograde flow rather than producing tension, and no forward advancement will occur. Our results support and expand on this model by presenting evidence that the dynamic assembly, growth, and disassembly of integrindependent point contacts depend on tightly balanced and localized Racl and RhoA activities.

It remains unclear how Racl is downregulated, but evidence in motile non-neuronal cells suggests that mechanical tension generates signals that reduce Racl activity. For example, when direct mechanical tension is applied to cells, Racl activity decreases, inhibiting further lamellipodial protrusion (Katsumi et al., 2002). Therefore, nascent point contacts induced by Rac1 may support tension, which in turn decreases Racl activity in a negative feedback loop. Furthermore, when tension was applied to cells uniaxially, protrusion was inhibited along the sides of the cell parallel to the direction of tension (Katsumi et al., 2002) accompanied by a redistribution of active Racl to sites perpendicular to the direction of tension, in effect mimicking the polarized cell morphology seen during directed migration. This raises 
the possibility that directionally applied tension (e.g., resulting from asymmetrically distributed point contacts) could act as a steering mechanism within growth cones. How tension reduces Racl activity is uncertain, but it may be through the activation of RhoA, which was shown to inhibit Racl in neurons (Li et al., 2002).

We also tested whether guidance cues known to signal through the Rho GTPases (for review, see Huber et al., 2003) influenced axon outgrowth by modulating growth cone point contact dynamics. We found that a low dose of Sema3A destabilized existing point contacts and inhibited new point contact formation similar to $\mathrm{CA}$ Rac1 expression. This result is consistent with previous studies showing that Sema3A activates Racl (Turner et al., 2004) and reduces integrin-dependent adhesion (Mikule et al., 2002; Serini et al., 2003). Although acute stimulation with LN also activated Rac1 (Fig. 7), this treatment initiated the rapid formation of multiple stable point contacts and accelerated neurite outgrowth. The opposing effects of LN and Sema3A may be attributable in part to different levels of active Rac1 before stimulation. On PDL, baseline Rac1 activity is low, whereas on LN, Racl activity may be near optimal so further increasing Rac1 may destabilize existing point contacts. This is consistent with previous studies showing that intermediate levels of active Racl promote neurite outgrowth (Kuhn et al., 1998) and directional persistence during cell migration (Pankov et al., 2005). In addition, it is likely that distinct signaling pathways activated by integrin and neuropilin/plexin receptors act independently to regulate point contact dynamics. Additional work is needed to determine the mechanisms involved in point contact destabilization downstream of Sema3A. It is possible that Sema3A may affect adhesion by controlling integrin receptor recycling. Sema3A has been shown to stimulate Rac1-dependent endocytosis (Fournier et al., 2000; Jurney et al., 2002), and increased integrin endocytosis can reduce cell adhesion (Proux-Gillardeaux et al., 2005). However, Sema3A may also directly control the affinity of integrin receptors for ECM ligands, as has been proposed for vascular endothelial cells (Serini et al., 2003).

It is noteworthy that a low dose of Sema3A was able to alter point contact dynamics without affecting changes in membrane protrusion as observed at higher concentrations (Fan et al., 1993; Fournier et al., 2000; Jurney et al., 2002). This result suggests that the effects of Sema3A on protrusion and adhesion can be distinct but may occur in parallel during full growth cone collapse. In addition, the low dose of Sema3A used in this study may be more

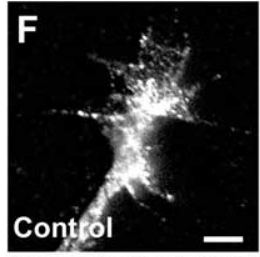
bars indicate SEM.
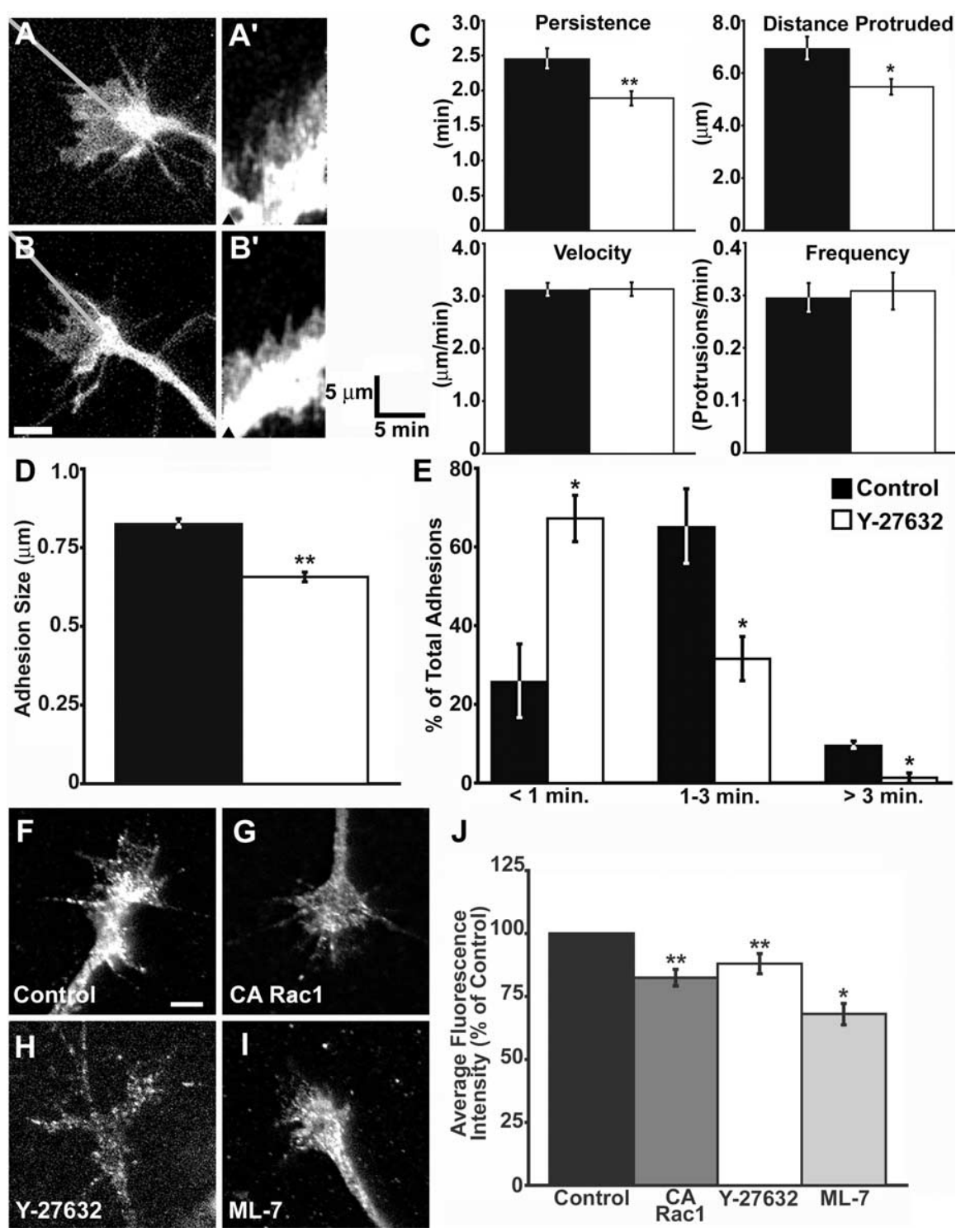

Figure 9. ROCK inhibition alters protrusion and point contact dynamics. $\boldsymbol{A}, \boldsymbol{B}, \mathrm{A}$ growth cone expressing paxillin-GFP imaged for $15 \mathrm{~min}$ at 10 s intervals before $(\boldsymbol{A})$ and $30 \mathrm{~min}$ after $(\boldsymbol{B})$ application of $25 \mu \mathrm{M}$ Y-27632 $(n=29)$. $\boldsymbol{A}^{\prime}, \boldsymbol{B}^{\prime}$, Kymographs were generated along the lines in $\boldsymbol{A}$ and $\boldsymbol{B}$. The arrowheads indicate the time points of the images in $\boldsymbol{A}$ and $\boldsymbol{B}$. Note that after ROCK inhibition, the contour of the kymograph becomes more jagged because of reduced protrusion persistence. $\boldsymbol{C}$, Quantification of protrusion persistence, distance, velocity, and frequency. Y-27632 causes a significant decrease in protrusion persistence and distance. $\boldsymbol{D}$, ROCK inhibition significantly decreases point contact size. Wild-type growth cones on LN were treated with $25 \mu \mathrm{M}$ Y-27632 (open bar; $n=36$ ) or control media (filled bar; $n=39$ ) for $30-45 \mathrm{~min}$, fixed, and stained with an antibody against paxillin. The lengths of identifiable point contacts were measured along their longest cord (see Materials and Methods). $\boldsymbol{E}$, Distribution of point contact lifetimes. Growth cones expressing paxillin-GFP were imaged for $15 \mathrm{~min}$ at $10 \mathrm{~s}$ intervals before (filled bar) and $30 \mathrm{~min}$ after (open bar) the addition of $25 \mu \mathrm{M}$ Y-27632 $(n=10)$. ROCK inhibition results in a greater proportion of transient point contacts. $\boldsymbol{F}-\mathbf{I}$, A phospho-specific (Ser19) antibody against MLC was used to label fixed growth cones expressing CA-Rac1 $(\mathbf{G} ; n=23)$ or treated with $25 \mu \mathrm{M} \mathrm{Y-27532}(\boldsymbol{H} ; n=33)$ or $10 \mu \mathrm{M} \mathrm{ML-7}(\boldsymbol{I} ; n=26)$, an inhibitor of myosin light chain kinase. All three manipulations decreased MLC phosphorylation relative to control $(\boldsymbol{F} ; n=67)$, suggesting reduced myosin-II activity. J, Quantification of p-MLC normalized to control. The average fluorescence intensity was measured within the entire growth cone using MetaMorph software. Scale bars, $5 \mu \mathrm{m}$. The calibration bars are as indicated. ${ }^{*} p<0.05$; $^{* *} p<0.001$. Error

physiological than the concentration that induces collapse, and the loss of growth cone adhesion could be an important component of repulsive turning in vivo. In fact, modulating growth cone adhesion may be a general mechanism used during axon pathfinding. Bonner and O'Connor (2001) showed that an interaction between LN and Tibial (Ti1) pioneer axons in the grasshopper embryo is required for growth cone steering in vivo. Changes 

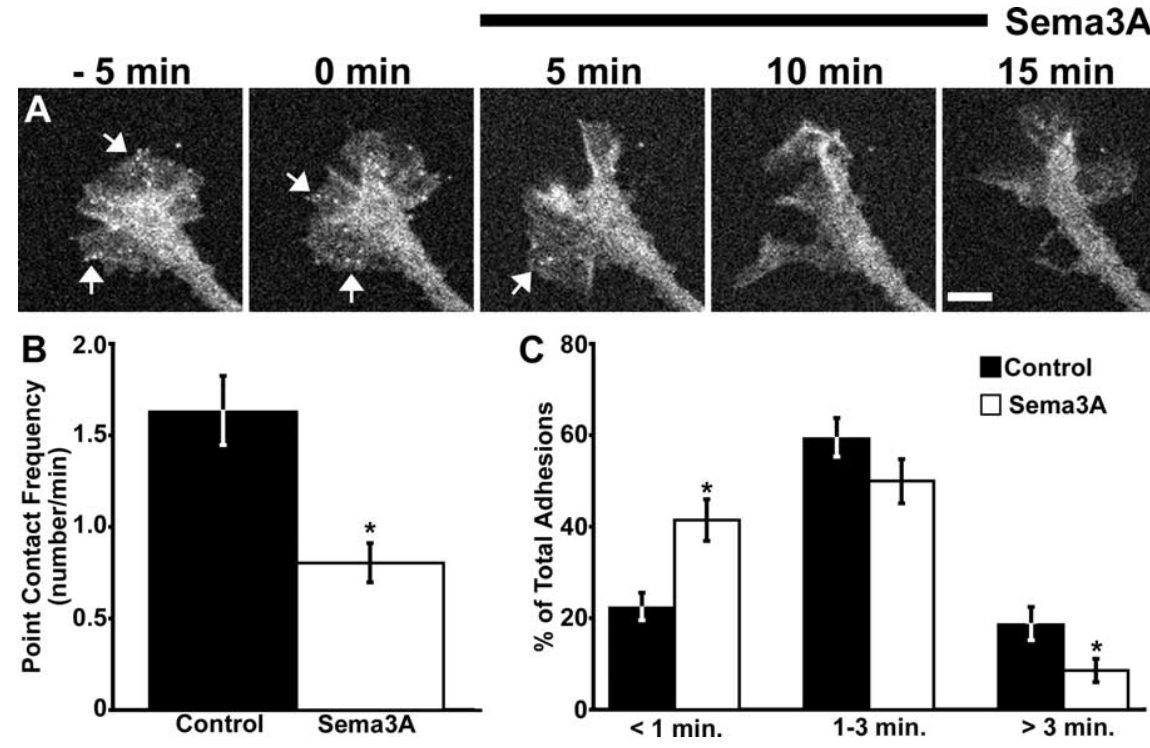

Figure 10. Sema3A inhibits point contact formation and stabilization. RGC growth cones expressing paxillin-GFP were imaged at 10 sintervals for $5-15 \mathrm{~min}$ before and $15 \mathrm{~min}$ after the addition of $400 \mathrm{ng} / \mathrm{ml} \mathrm{Sema3A}(n=15)$. A, A RGC growth cone expressing paxillin-GFP at the indicated times before and after the addition of $400 \mathrm{ng} / \mathrm{ml} \mathrm{Sema3A}$. The arrows point to representative point contacts. Note that there are fewer point contacts within $5 \mathrm{~min}$ of Sema3A addition and that the growth cone changes shape but does not collapse or retract. See supplemental material (available at www.jneurosci.org) for the movie corresponding to this growth cone (movie 8). $\boldsymbol{B}$, Comparison of the frequency of new point contact formation before and after Sema3A addition shows that point contacts form at a significantly reduced frequency after Sema3A addition. $C$, Analysis of point contact lifetimes shows that after Sema3A addition, a greater proportion of point contacts are transient with correspondingly fewer stable contacts compared with pretreatment control. Scale bar $5 \mu \mathrm{m} .{ }^{*} p<0.05$. Error bars indicate SEM.

in ECM composition have also been proposed to underlie RGC axon pathfinding (Mann et al., 2004). In addition to Sema3A, other guidance cues have been shown to affect integrindependent adhesion including Ephrin-A1 (Miao et al., 2000), Slit (Stevens and Jacobs, 2002), Semaphorin 7A (Pasterkamp et al., 2003), and Netrin (Yebra et al., 2003). Illustrating a direct role for modulating integrin-dependent adhesion in axon pathfinding, whether through the composition of the ECM, the actions soluble guidance cues, or a combination of the two, is an important future goal in this area.

\section{References}

Akiyama SK, Yamada SS, Chen WT, Yamada KM (1989) Analysis of fibronectin receptor function with monoclonal antibodies: roles in cell adhesion, migration, matrix assembly, and cytoskeletal organization. J Cell Biol 109:863-875.

Amano M, Ito M, Kimura K, Fukata Y, Chihara K, Nakano T, Matsuura Y, Kaibuchi K (1996) Phosphorylation and activation of myosin by Rhoassociated kinase (Rho-kinase). J Biol Chem 271:20246-20249.

Beningo KA, Dembo M, Kaverina I, Small JV, Wang YL (2001) Nascent focal adhesions are responsible for the generation of strong propulsive forces in migrating fibroblasts. J Cell Biol 153:881-888.

Bokoch GM (2003) Biology of the p21-activated kinases. Annu Rev Biochem 72:743-781.

Bonner J, O'Connor TP (2001) The permissive cue laminin is essential for growth cone turning in vivo. J Neurosci 21:9782-9791.

Campbell DS, Regan AG, Lopez JS, Tannahill D, Harris WA, Holt CE (2001) Semaphorin $3 \mathrm{~A}$ elicits stage-dependent collapse, turning, and branching in Xenopus retinal growth cones. J Neurosci 21:8538-8547.

Chrzanowska-Wodnicka M, Burridge K (1996) Rho-stimulated contractility drives the formation of stress fibers and focal adhesions. J Cell Biol 133:1403-1415.

Del Pozo MA, Kiosses WB, Alderson NB, Meller N, Hahn KM, Schwartz MA (2002) Integrins regulate GTP-Rac localized effector interactions through dissociation of Rho-GDI. Nat Cell Biol 4:232-239.

Fan J, Mansfield SG, Redmond T, Gordon-Weeks PR, Raper JA (1993) The
Sema3A

organization of F-actin and microtubules in growth cones exposed to a brain-derived collapsing factor. J Cell Biol 121:867-878.

Fournier AE, Nakamura F, Kawamoto S, Goshima Y, Kalb RG, Strittmatter SM (2000) Semaphorin3A enhances endocytosis at sites of receptor-F-actin colocalization during growth cone collapse. J Cell Biol 149:411-422.

Geiger B, Bershadsky A, Pankov R, Yamada KM (2001) Transmembrane crosstalk between the extracellular matrix-cytoskeleton crosstalk. Nat Rev Mol Cell Biol 2:793-805.

Giannone G, Ronde P, Gaire M, Haiech J, Takeda K (2002) Calcium oscillations trigger focal adhesion disassembly in human U87 astrocytoma cells. J Biol Chem 277:26364-26371.

Gomez TM, Roche FK, Letourneau PC (1996) Chick sensory neuronal growth cones distinguish fibronectin from laminin by making substratum contacts that resemble focal contacts. J Neurobiol 29:18-34.

Gomez TM, Robles E, Poo M, Spitzer NC (2001) Filopodial calcium transients promote substrate-dependent growth cone turning. Science 291:1983-1987.

Gomez TM, Harrigan D, Henley J, Robles E (2003) Working with Xenopus spinal neurons in live cell culture. Methods Cell Biol 71:129-156.

Hinz B, Alt W, Johnen C, Herzog V, Kaiser HW (1999) Quantifying lamella dynamics of cultured cells by SACED, a new computerassisted motion analysis. Exp Cell Res 251:234-243.

Huber AB, Kolodkin AL, Ginty DD, Cloutier JF (2003) Signaling at the growth cone: ligandreceptor complexes and the control of axon growth and guidance. Annu Rev Neurosci 26:509-563.

Ivins JK, Parry MK, Long DA (2004) A novel cAMP-dependent pathway activates neuronal integrin function in retinal neurons. J Neurosci 24:1212-1216.

Jalink K, van Corven EJ, Hengeveld T, Morii N, Narumiya S, Moolenaar WH (1994) Inhibition of lysophosphatidate- and thrombin-induced neurite retraction and neuronal cell rounding by ADP ribosylation of the small GTP-binding protein Rho. J Cell Biol 126:801-810.

Jurney WM, Gallo G, Letourneau PC, McLoon SC (2002) Rac1-mediated endocytosis during ephrin-A2- and semaphorin 3A-induced growth cone collapse. J Neurosci 22:6019-6028.

Katsumi A, Milanini J, Kiosses WB, del Pozo MA, Kaunas R, Chien S, Hahn KM, Schwartz MA (2002) Effects of cell tension on the small GTPase Rac. J Cell Biol 158:153-164.

Kimura K, Ito M, Amano M, Chihara K, Fukata Y, Nakafuku M, Yamamori B, Feng J, Nakano T, Okawa K, Iwamatsu A, Kaibuchi K (1996) Regulation of myosin phosphatase by Rho and Rho-associated kinase (Rho-kinase). Science 273:245-248.

Kuhn TB, Brown MD, Bamburg JR (1998) Rac1-dependent actin filament organization in growth cones is necessary for betal-integrin-mediated advance but not for growth on poly-D-lysine. J Neurobiol 37:524-540.

Li Z, Aizenman CD, Cline HT (2002) Regulation of rho GTPases by crosstalk and neuronal activity in vivo. Neuron 33:741-750.

Luo L (2000) Rho GTPases in neuronal morphogenesis. Nat Rev Neurosci 1:173-180.

Mann F, Harris WA, Holt CE (2004) New views on retinal axon development: a navigation guide. Int J Dev Biol 48:957-964.

Miao H, Burnett E, Kinch M, Simon E, Wang B (2000) Activation of EphA2 kinase suppresses integrin function and causes focal-adhesion-kinase dephosphorylation. Nat Cell Biol 2:62-69.

Mikule K, Gatlin JC, de la Houssaye BA, Pfenninger KH (2002) Growth cone collapse induced by semaphorin $3 \mathrm{~A}$ requires 12/15-lipoxygenase. J Neurosci 22:4932-4941.

Mitchison T, Kirschner M (1988) Cytoskeletal dynamics and nerve growth. Neuron 1:761-772. 
Nakamura T, Aoki K, Matsuda M (2005) FRET imaging in nerve growth cones reveals a high level of RhoA activity within the peripheral domain. Brain Res Mol Brain Res 139:277-287.

Ng J, Nardine T, Harms M, Tzu J, Goldstein A, Sun Y, Dietzl G, Dickson BJ, Luo L (2002) Rac GTPases control axon growth, guidance and branching. Nature 416:442-447.

Niederost B, Oertle T, Fritsche J, McKinney RA, Bandtlow CE (2002) Nogo-A and myelin-associated glycoprotein mediate neurite growth inhibition by antagonistic regulation of RhoA and Rac1. J Neurosci 22:10368-10376.

Nieuwkoop PD, Faber J (1994) Normal table of Xenopus laevis (Daudin). New York: Garland.

Nobes CD, Hall A (1995) Rho, rac, and cdc42 GTPases regulate the assembly of multimolecular focal complexes associated with actin stress fibers, lamellipodia, and filopodia. Cell 81:53-62.

Pankov R, Endo Y, Even-Ram S, Araki M, Clark K, Cukierman E, Matsumoto K, Yamada KM (2005) A Rac switch regulates random versus directionally persistent cell migration. J Cell Biol 170:793-802.

Pasterkamp RJ, Peschon JJ, Spriggs MK, Kolodkin AL (2003) Semaphorin 7A promotes axon outgrowth through integrins and MAPKs. Nature 424:398-405.

Proux-Gillardeaux V, Gavard J, Irinopoulou T, Mege RM, Galli T (2005) Tetanus neurotoxin-mediated cleavage of cellubrevin impairs epithelial cell migration and integrin-dependent cell adhesion. Proc Natl Acad Sci USA 102:6362-6367.

Renaudin A, Lehmann M, Girault J, McKerracher L (1999) Organization of point contacts in neuronal growth cones. J Neurosci Res 55:458-471.

Ridley AJ, Schwartz MA, Burridge K, Firtel RA, Ginsberg MH, Borisy G, Parsons JT, Horwitz AR (2003) Cell migration: integrating signals from front to back. Science 302:1704-1709.

Rottner K, Hall A, Small JV (1999) Interplay between Rac and Rho in the control of substrate contact dynamics. Curr Biol 9:640-648.

Ruchhoeft ML, Ohnuma S, McNeill L, Holt CE, Harris WA (1999) The neuronal architecture of Xenopus retinal ganglion cells is sculpted by rhofamily GTPases in vivo. J Neurosci 19:8454-8463.

Sanders LC, Matsumura F, Bokoch GM, de Lanerolle P (1999) Inhibition of myosin light chain kinase by p21-activated kinase. Science 283:2083-2085

Serini G, Valdembri D, Zanivan S, Morterra G, Burkhardt C, Caccavari F, Zammataro L, Primo L, Tamagnone L, Logan M, Tessier-Lavigne M, Taniguchi M, Puschel AW, Bussolino F (2003) Class 3 semaphorins control vascular morphogenesis by inhibiting integrin function. Nature 424:391-397.

Shamah SM, Lin MZ, Goldberg JL, Estrach S, Sahin M, Hu L, Bazalakova M, Neve RL, Corfas G, Debant A, Greenberg ME (2001) EphA receptors regulate growth cone dynamics through the novel guanine nucleotide exchange factor ephexin. Cell 105:233-244.

Shekarabi M, Kennedy TE (2002) The netrin-1 receptor DCC promotes filopodia formation and cell spreading by activating Cdc42 and Rac1. Mol Cell Neurosci 19:1-17.

Song H, Ming G, He Z, Lehmann M, McKerracher L, Tessier-Lavigne M, Poo M (1998) Conversion of neuronal growth cone responses from repulsion to attraction by cyclic nucleotides. Science 281:1515-1518.

Stevens A, Jacobs JR (2002) Integrins regulate responsiveness to slit repellent signals. J Neurosci 22:4448-4455.

Suter DM, Forscher P (2000) Substrate-cytoskeletal coupling as a mechanism for the regulation of growth cone motility and guidance. J Neurobiol 44:97-113.

Suter DM, Errante LD, Belotserkovsky V, Forscher P (1998) The Ig superfamily cell adhesion molecule, apCAM, mediates growth cone steering by substrate-cytoskeletal coupling. J Cell Biol 141:227-240.

Swiercz JM, Kuner R, Behrens J, Offermanns S (2002) Plexin-B1 directly interacts with PDZ-RhoGEF/LARG to regulate RhoA and growth cone morphology. Neuron 35:51-63.

Turner LJ, Nicholls S, Hall A (2004) The activity of the plexin-A1 receptor is regulated by Rac. J Biol Chem 279:33199-33205.

van Leeuwen FN, van Delft S, Kain HE, van der Kammen RA, Collard JG (1999) Rac regulates phosphorylation of the myosin-II heavy chain, actinomyosin disassembly and cell spreading. Nat Cell Biol 1:242-248.

Varnum-Finney B, Reichardt LF (1994) Vinculin-deficient PC12 cell lines extend unstable lamellipodia and filopodia and have a reduced rate of neurite outgrowth. J Cell Biol 127:1071-1084.

Vastrik I, Eickholt BJ, Walsh FS, Ridley A, Doherty P (1999) Sema3Ainduced growth-cone collapse is mediated by Racl amino acids 17-32. Curr Biol 9:991-998.

Wu KY, Hengst U, Cox LJ, Macosko EZ, Jeromin A, Urquhart ER, Jaffrey SR (2005) Local translation of RhoA regulates growth cone collapse. Nature 436:1020-1024.

Yebra M, Montgomery AM, Diaferia GR, Kaido T, Silletti S, Perez B, Just ML, Hildbrand S, Hurford R, Florkiewicz E, Tessier-Lavigne M, Cirulli V (2003) Recognition of the neural chemoattractant Netrin-1 by integrins alpha6beta 4 and alpha3beta 1 regulates epithelial cell adhesion and migration. Dev Cell 5:695-707. 